\title{
Assessing real-world vaccine effectiveness against severe forms of SARS-CoV-2 infection from routine surveillance data in Switzerland
}

\author{
Nanina Anderegg (1,2), Christian L. Althaus (1), Samuel Colin (2), Anthony \\ Hauser (1), Anne Laube (2), Mirjam Mäusezahl (2), Moritz Wagner (2), \\ Biagio Zaffora (2), Julien Riou $\left(1,2,{ }^{*}\right)$
}

(1) Institute of Social and Preventive Medicine, University of Bern, Switzerland. (2) Federal Office of Public Health, Switzerland. *Corresponding author: julien.riou@ispm.unibe.ch

\section{Summary}

Background. In Switzerland, SARS-CoV-2 vaccination campaigns started early 2021. Vaccine coverage reached 65\% of the population in December 2021, mostly using mRNA vaccines from Moderna and Pfizer-BioNtech. Simultaneously, the proportion of vaccinated among COVID-19-related hospitalizations and deaths rose, creating some confusion in the general population. We aim to assess vaccine effectiveness against severe forms of SARSCoV-2 infection using routine surveillance data on the vaccination status of COVID-19related hospitalizations and deaths and data on vaccination coverage in Switzerland.

Methods. We consider all routine surveillance data on COVID-19-related hospitalizations and deaths received at the Swiss Federal Office of Public Health from 1 July 2021 to 1 December 2021. We estimate the relative risk of COVID-19 related hospitalization or death for non-fully vaccinated compared to fully vaccinated individuals, adjusted for the dynamics of vaccination coverage over time, by age and location. We stratify the analysis by age group and by calendar month. We assess variations in the relative risk of hospitalization associated with the time since vaccination.

Results. We include a total of 5,948 COVID-19-related hospitalizations of which 1,245 (21\%) were fully vaccinated, and a total of 739 deaths of which 259 (35\%) were fully vaccinated. We find that the relative risk of COVID-19 related hospitalization is 12.5 (95\%CI: 11.7 to 13.4 ) times higher for non-fully vaccinated than for fully vaccinated individuals. This translates into a vaccine effectiveness against hospitalization of $92.0 \%$ (95\%CI: 91.4 to 92.5\%). Vaccine effectiveness against death is estimated to $90.3 \%$ (95\%CI: 88.6 to $91.8 \%$ ). Effectiveness appears comparatively lower in age groups over 70 and during the months of October and November 2021. We also find evidence of a decrease in vaccine effectiveness against hospitalization for individuals vaccinated for 25 weeks or more, but this decrease only appears in age groups below 70.

Conclusions. The observed proportions of vaccinated among COVD-19-related hospitalizations and deaths in Switzerland are compatible with a high effectiveness of mRNA vaccines from Moderna and Pfizer-BioNtech against hospitalization and death in all 
age groups. Effectiveness appears comparatively lower in older age groups, suggesting the importance of booster vaccinations. We find inconclusive evidence that vaccine effectiveness is waning over time. Repeated analyses will be able to better assess waning and the effect of boosters.

\section{Introduction}

The continuous assessment of vaccine efficacy and effectiveness against SARS-CoV-2 is critically important for informing national vaccination campaigns and the public health response against the COVID-19 pandemic. Randomized controlled trials (RCT) are the gold standard to estimate vaccine efficacy against symptomatic infection, hospitalization and death. Several RCTs have reported high levels of efficacy for several SARS-CoV-2 vaccines $[1,2]$. For technical and ethical reasons, RCTs have limitations when it comes to estimate vaccine effectiveness in real world conditions and over longer periods of time[3,4]. Even though not ideal in terms of potential bias, observational data can be used to estimate vaccine effectiveness. When rich, longitudinal data is available (e.g. insurance data or cohort studies), it becomes possible to directly estimate and compare the risk of symptomatic infection, hospitalization or death in vaccinated and non-vaccinated individuals (adjusting for key characteristics) [5,6]. When the vaccination status of SARS-CoV-2-negative controls is collected, a test-negative design can be used $[7,8]$.

Routine surveillance data often does not include any follow-up or control group. In Switzerland, routine surveillance data on COVID-19 only contains detailed information on reported confirmed cases, hospitalizations and deaths. Observing the proportion of vaccinated in surveillance reports can however be misleading as it is highly dependent on vaccination coverage [9]. Vaccination coverage can be heterogeneous and can vary by time, location and other characteristics, first of all age. In Switzerland, SARS-CoV-2 vaccination campaigns started in early 2021, first focusing on vulnerable groups (i.e. aged above 65 with comorbid conditions), then being gradually extended to younger age groups. Until May 2021, vaccination was roughly equally done with vaccines from Moderna and PfizerBioNtech, but from this point onward Moderna was more commonly used. Vaccination with Johnson-Johnson only started in October 2021.

In this study, we use a reformulation of the screening method $[10,11]$ to estimate vaccine effectiveness against severe forms of SARS-CoV-2 infection in real-world settings from routine surveillance data in Switzerland, accounting for the levels of vaccination coverage by week, age group and location. We also assess the variation in vaccine effectiveness by age, vaccine type, by calendar time and - importantly - by time since vaccination.

\section{Methods}

\section{Setting and data}

We consider all routine surveillance data on COVID-19-related hospitalizations and deaths received at the Federal Office of Public Health until 1 December 2021. This data includes vaccination status at the individual level, which accounts for the type of vaccine, the 
number of doses, and the existence of a previous positive test. Individuals are considered fully vaccinated if they received either two doses of Moderna or Pfizer-BioNtech vaccine, one dose of Johnson-Johnson vaccine, or one dose of Moderna or Pfizer-BioNtech vaccine with a previous positive test. Individuals are considered partially vaccinated if they received just one dose of Moderna or Pfizer-BioNtech without a previous positive test. Individuals are considered non-vaccinated if they reported having not received any dose.

We only include COVID-19-related hospitalizations and deaths from 1 July 2021. Indeed, the proportion of missing vaccination status among hospitalizations and deaths is high (30$60 \%$ ) during the early months of 2021 , but rapidly decreases to around $10 \%-15 \%$ from the month of July 2021 onward (supplementary Figure S1).

We treat missing information on the vaccination status as follows. For the baseline analysis, we define as non-fully vaccinated individuals who reported to be either non-vaccinated or partially-vaccinated, and exclude individuals with missing vaccination status. This assumes that vaccination status is missing at random (i.e. non-fully vaccinated individuals are equally likely to have missing vaccination status as fully vaccinated individuals). We then conduct several sensitivity analyses. First, we impute missing vaccination status based on age group, canton, week, and vaccination coverage using multiple imputation with chained equations[12]. Second, we assume that all individuals with missing vaccination status are non-fully vaccinated ("Best case scenario"). Third, we assume that all individuals with missing vaccination status and all partially-vaccinated are fully-vaccinated ("Worst case scenario").

\section{Stastistical model}

We use a statistical model to assess the relative risk of hospitalization $(R R)$ among non-fully vaccinated individuals $(\bar{V})$ compared to fully vaccinated $(V)$ :

$$
R R=\frac{\operatorname{Pr}(H \mid \bar{V})}{\operatorname{Pr}(H \mid V)}
$$

where $\operatorname{Pr}(H \mid \bar{V})$ refers to the probability of hospitalization given non-fully vaccinated status and $\operatorname{Pr}(H \mid V)$ to the probability of hospitalization given fully vaccinated status. This models allows us to estimate $R R$ while accounting for the dynamics of vaccination coverage over time, by age group and canton. The approach, which is detailed in the supplementary appendix, is equivalent to the screening method [10]. Briefly, we consider the expected probability that a hospitalized individual is vaccinated $(\operatorname{Pr}(V \mid H))$ given the vaccination coverage at the time of hospitalization in the same age group and canton $(P(V))$, and given the value of $R R$ from the following equation:

$$
\operatorname{Pr}(V \mid H)=\frac{\operatorname{Pr}(V)}{\operatorname{Pr}(V)+R R(1-\operatorname{Pr}(V))} .
$$

We estimate the $R R$ by comparing the expected probability $P(V \mid H)$ to the actual vaccination status of every individual using a Bernoulli likelihood within a maximum likelihood framework. The $R R$ can also be expressed as a relative risk reduction $(1-1 / R R)$. This last quantity is closely related to vaccine effectiveness against hospitalization if we make the 
assumption that all the other factors influencing the risk of COVID-19 hospitalization (e.g. behavior or exposure) are independent from the vaccination status.

This model also applies to vaccine effectiveness against death, and can be extended to assess variations in vaccine effectiveness across different stratification groups. We consider stratification by age group and calendar month. We also extend the approach to a situation where we compare more than the initial two categories (fully vaccinated or not) using a generalization of the equations above (supplementary appendix). This allows to assess variations in the $R R$ associated with time since vaccination in three groups (vaccinated for up to 12 weeks, for 13 to 24 weeks, or for 25 weeks or more). We restrict this analysis to fully vaccinated individuals with non-missing time since vaccination, assuming that this variable is missing at random. We also stratify by age group and by vaccine type (restricted to Moderna or Pfizer-BioNtech).

The code is available at https://github.com/jriou/vaccine_effectiveness, together with a simulation study validating the statistical approach. As data contains sensitive information at the individual level, it is only available on motivated request to the Swiss Federal Office of Public Health.

\section{Results}

From 1 July 2021 to 1 December 2021 we include a total of 5,948 hospitalizations and 739 deaths (Table 1). Among all hospitalizations and deaths the proportion of fully-vaccinated has increased over time to around 40\% (Figure 1A). During the same period, vaccination coverage in the population has increased to $65.7 \%$, with important differences across age groups (Figure 1B). There are also geographical differences in vaccination coverage and the type of vaccine used (Figure 1C), highlighting the importance of accounting for vaccination coverage by time, age group and location. 

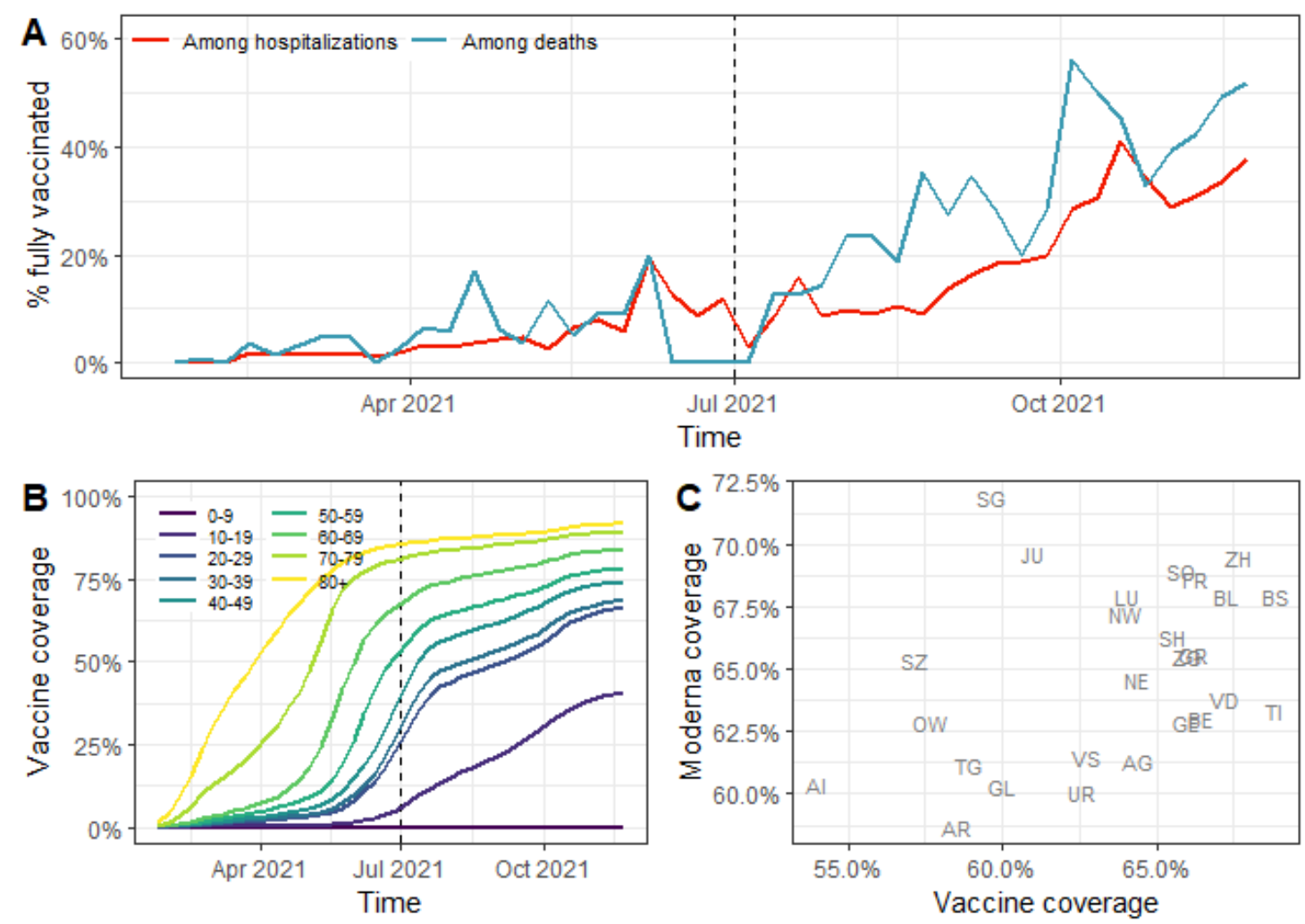

Figure 1. (A) Proportion of hospitalized and deceased COVID-19 patients reported as fully vaccinated among all reported COVID-19-related hospitalizations and deaths. The dashed vertical line corresponds to the starting date of the analysis. (B) Evolution of SARS-CoV-2 vaccination coverage in the population by age group. (C) Cantonal differences regarding the overall SARS-CoV-2 vaccination coverage and the proportion of fully vaccinated individuals having received Moderna in the population (as of one week before the end of the study period).

Of hospitalized individuals, 1,245 (21\%) are reported as being fully vaccinated (Table 1). This number is 259 (35\%) for deaths. Vaccination status is missing for $834(14 \%)$ of hospitalizations and $98(13 \%)$ of deaths. The age distribution of hospitalized patients is shifted towards older age groups, with 1,221 (21\%) being 80 and older. This is even more pronounced for deceased patients, with 464 (63\%) being 80 and older. Almost all individuals received the mRNA vaccines of Moderna or Pfizer-BioNtech. Among fully vaccinated, $373(30 \%)$ of hospitalizations and $133(51 \%)$ of deaths have been vaccinated for 25 weeks or more. Information about time since vaccination is missing for 584 (47\%) of hospitalizations and 83 (32\%) of deaths. 
Table 1. Description of included COVID-19-related hospitalized and deceased persons from 1 July 2021 to 1 December 2021.

\begin{tabular}{|c|c|c|c|}
\hline & & Hospitalizations & Deaths \\
\hline & Total & $5,948(100 \%)$ & 739 (100\%) \\
\hline \multirow[t]{4}{*}{ Vaccination status } & Fully vaccinated & $1,245(21 \%)$ & $259(35 \%)$ \\
\hline & Partially vaccinated & $69(1 \%)$ & $6(1 \%)$ \\
\hline & Not vaccinated & $3,800(64 \%)$ & $376(51 \%)$ \\
\hline & Missing & $834(14 \%)$ & 98 (13\%) \\
\hline \multirow[t]{9}{*}{ Age [years] } & $0-9$ & $115(2 \%)$ & $0(0 \%)$ \\
\hline & $10-19$ & $57(1 \%)$ & $0(0 \%)$ \\
\hline & $20-29$ & $224(4 \%)$ & $1(0 \%)$ \\
\hline & $30-39$ & $516(9 \%)$ & $4(1 \%)$ \\
\hline & $40-49$ & $773(13 \%)$ & $8(1 \%)$ \\
\hline & $50-59$ & $1,050(18 \%)$ & $44(6 \%)$ \\
\hline & $60-69$ & 999 (17\%) & $80(11 \%)$ \\
\hline & $70-79$ & $993(17 \%)$ & $138(19 \%)$ \\
\hline & $80+$ & $1,221(21 \%)$ & $464(63 \%)$ \\
\hline \multirow[t]{4}{*}{$\begin{array}{l}\text { Vaccine type (among fully } \\
\text { vaccinated) }\end{array}$} & Moderna & $310(25 \%)$ & $54(21 \%)$ \\
\hline & Pfizer-BioNtech & 357 (29\%) & $120(46 \%)$ \\
\hline & Johnson-Johnson & $14(1 \%)$ & $0(0 \%)$ \\
\hline & Missing & $564(45 \%)$ & $85(33 \%)$ \\
\hline \multirow[t]{4}{*}{$\begin{array}{l}\text { Weeks since vaccination } \\
\text { (among fully vaccinated) }\end{array}$} & 0 to 12 weeks & $88(7 \%)$ & $17(7 \%)$ \\
\hline & 13 to 24 weeks & $200(16 \%)$ & $26(10 \%)$ \\
\hline & $25+$ weeks & $373(30 \%)$ & $133(51 \%)$ \\
\hline & Missing & $584(47 \%)$ & $83(32 \%)$ \\
\hline
\end{tabular}

Accounting for vaccination coverage by week, age group and canton, we find that the $R R$ of hospitalization without full vaccination compared to full vaccination is 12.5 (95\% $\mathrm{CI}$ : 11.7 to 13.4) (Figure 2A, supplementary table S1). This corresponds to a relative risk reduction against hospitalization (or vaccine effectiveness) of $92.0 \%$ (95\%CI: 91.4 to $92.5 \%$ ). Results from sensitivity analyses with alternative handling of missing vaccination status range between $R R=6.6$ (95\%CI: 6.2 to 7.0 ) and 15.7 (95\%CI: 14.7 to 16.8). The $R R$ of 
hospitalization decreases in older age groups. This could be linked to a weaker immune response in older people. It could also be indirect evidence of waning, as older age groups were vaccinated first. We also find a decrease of the $R R$ of hospitalization over time, which also constitutes indirect evidence of waning. When stratifying by both age group and month, we find evidence for a decrease in $R R$ in October and November 2021 in age groups 70-79 and 80+, but inconclusive evidence for other age groups (supplementary Figure S3).

The $R R$ of death without full vaccination compared to full vaccination is 10.4 (95\%CI: 8.8 to 12.2) (Figure $2 \mathrm{~B}$, supplementary table $\mathrm{S} 2$ ), corresponding to a relative risk reduction against death of $90.3 \%$ (95\%CI: 88.6 to $91.8 \%$ ). The $R R$ of death decreases for older ages and over time, which again constitutes indirect evidence of waning. However, the small number of deaths leads to imprecise estimates with large confidence intervals. 

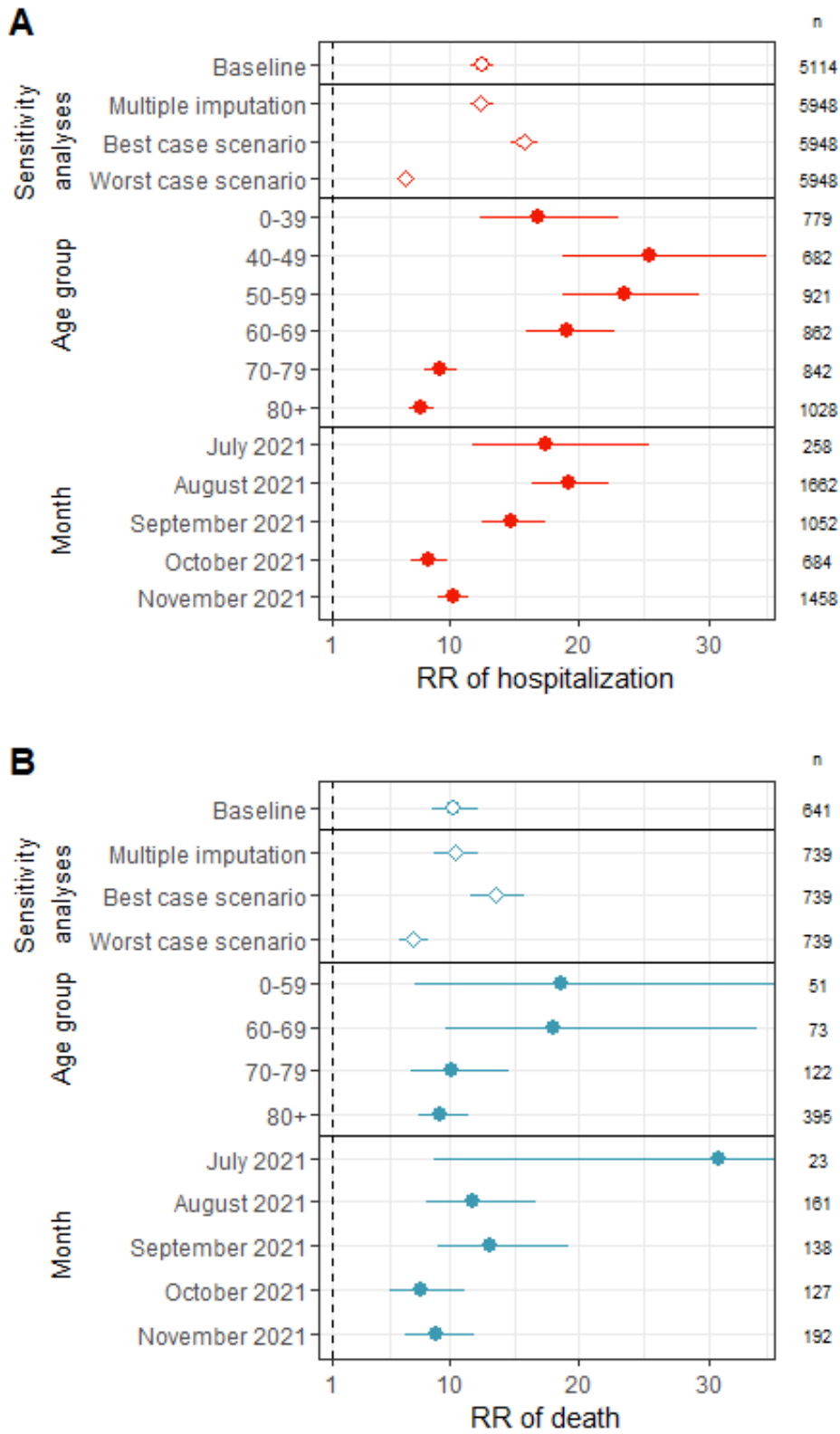

Figure 2. (A) Relative risk of COVID-19-related hospitalization for non-vaccinated individuals compared to vaccinated individuals in the baseline analysis, in three sensitivity analyses, by age group, and by month. (B) Relative risk of death for non-vaccinated individuals compared to vaccinated individuals in the baseline analysis, in three sensitivity analyses, by age group, and by month. Numbers correspond to sample sizes (n).

To study direct evidence of waning, we restrict analyses only to fully vaccinated individuals with information about time since vaccination. The outcome of interest is now the relative change in the $R R$ of hospitalization compared to the reference group of individuals vaccinated for up to 12 weeks. Compared to the reference group, the $R R$ of hospitalization does not change when the time since vaccination is between 13 and 24 weeks, but increases by 1.5 (95\%CI: 1.1 to 2.1 ) when the time since vaccination is above 25 weeks (Figure $3 \mathrm{~A}$ ). When stratifying by age group, this increase in the $R R$ of hospitalization 25 weeks after 
vaccination is only significant in the age groups $0-59$ (4.0 (95\%CI: 2.0 to 7.8)) and 60-69 (2.9 (95\%CI: 1.4 to 5.7)), but not in age groups 70-79 (1.6 (95\%CI: 0.9 to 2.8$)$ ) and $80+(0.7$ (95\%CI: 0.5 to 1.2 ), Figure 3B). When stratifying by vaccine type, the increase in $R R$ of hospitalization appears significant for both Pfizer-BioNtech (1.8 (95\%CI: 1.1 to 3.0)) and Moderna (2.4 (95\%CI: 1.4 to 4.0), Figure 3C).
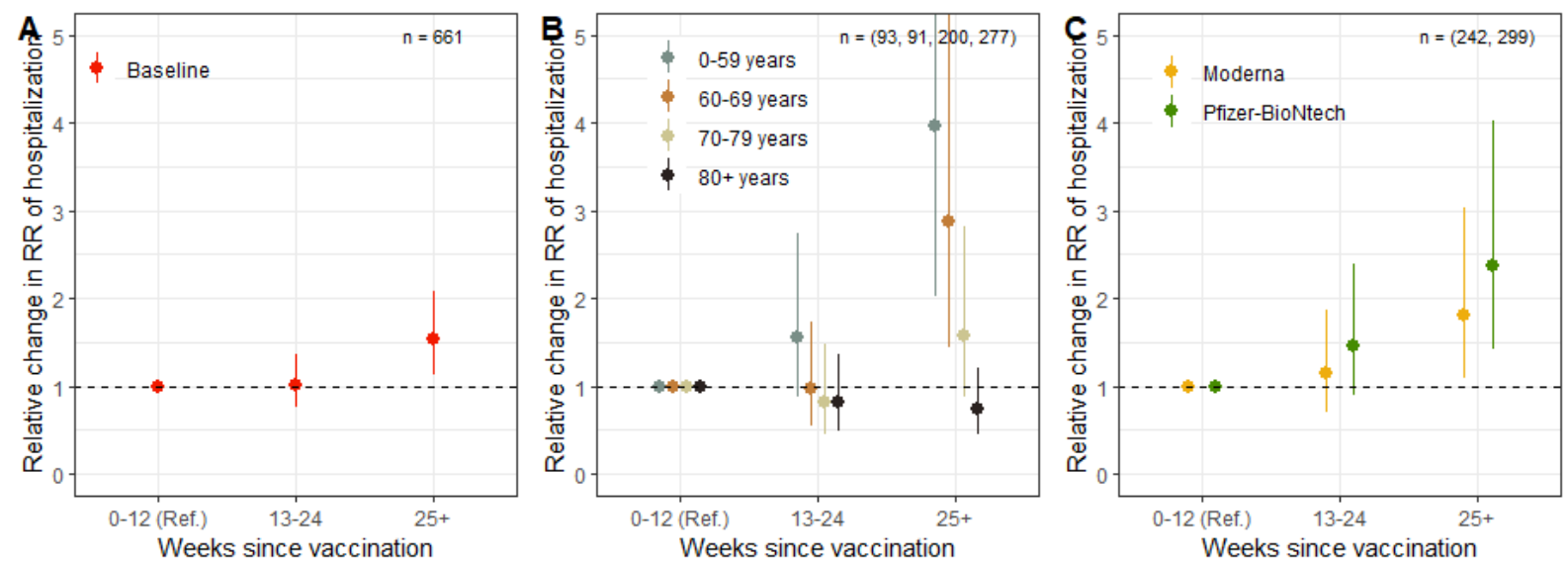

Figure 3. Change in the relative risk of COVID-19-related hospitalization depending on the time since vaccination in weeks (reference is 0 to 12 weeks), overall (panel A), by age group (panel B), and by vaccine type (panel C). Numbers correspond to sample sizes (n).

\section{Discussion}

Using surveillance data on COVID-19-related hospitalizations and deaths from Switzerland between 1 July 2021 to 1 December 2021 and accounting for the dynamics of vaccination coverage over time, by age and location, we estimate that non-fully vaccinated individuals have 12.5 times the risk of hospitalization and 10.4 times the risk of death compared to fully vaccinated individuals. This corresponds to a vaccine effectiveness of $92 \%$ against hospitalization and $90.3 \%$ against death. This is in agreement with other studies about vaccine effectiveness of mRNA vaccines $[13,14,15,16]$. Note that Delta was the dominant SARS-CoV-2 variant in Switzerland during this time period.

We also investigate the potential waning of vaccine effectiveness over time. We find evidence of a lower vaccine effectiveness in age groups above 70, which could be caused by a weaker immune response, but could also be interpreted as an indirect evidence of waning, as older age groups were vaccinated first. Similarly, we find a decrease in vaccine effectiveness in the months of October and November 2021, which also constitutes indirect evidence of waning. Directly investigating the variation in vaccine effectiveness by time since vaccination, we don't find evidence of a reduction of vaccine effectiveness up to 24 weeks after vaccination. From 25 weeks after vaccination, vaccine effectiveness appears to be moderately reduced (increase in the $R R$ of hospitalization by 1.5). To give a sense of the effect size, this value of 1.5 would correspond to a reduction of vaccine effectiveness from $92 \%$ to $88 \%$. 
On a closer look, this reduction of vaccine effectiveness among people vaccinated for more than 25 weeks does not appear consistently across age groups. It is only visible in the age groups below 70, to a lesser extent in the age group 70-79, but not in the age group 80+. This pattern could reflect an actual faster waning of immunity in younger individuals [17], although an opposite effect with a faster waning at older age ("immunosenescence") has also been described [18]. It could also be due to a confounding effect, whereby individuals younger than 70 with comorbidities are more likely to have been vaccinated for a longer time (as they were prioritized in the early stages of the vaccination campaign) and are also more likely to be hospitalized upon infection with SARS-CoV-2. Confounding by occupation could also play a role, as for instance healthcare workers are both more likely to have received vaccination early and more likely to be exposed to SARS-CoV-2. In any case, this apparent decrease of vaccine effectiveness in age groups below 70 has to be put in relation with a higher baseline vaccine effectiveness.

Looking at vaccine types, the reduction in vaccine effectiveness is apparent for both Moderna and Pfizer-BioNtech. We thus find some direct but inconclusive evidence of a moderate waning of mRNA vaccine effectiveness against hospitalization after 25 weeks, which is in agreement with data from Israel [19], Qatar [20] and New York state [21]. However, our findings are in contrast with other studies that showed considerably faster waning among older individuals after more than 6 months [22]. Of note, the direct comparison of vaccine types suggests that Pfizer-BioNtech is associated with a slightly higher $R R$ of hospitalization than Moderna (relative change of 1.8 (95\%CI: 1.5 to 2.1), supplementary Figure S4), as was shown in previous studies [14].

This study has some strengths and limitations. Applicable without control group or long follow-up times, our approach uses individual data and reverse conditionality to estimate the $R R$ of hospitalization or death for non-fully vaccinated compared to fully vaccinated, taking into account the dynamics of vaccination coverage by age group and location. This quantity can be estimated using different types of stratification, and is closely related to vaccine effectiveness. Our approach relies on several assumptions. In order to interpret the $R R$ in terms of vaccine effectiveness, we assume that, within a population of the same age group, in the same location and the same time frame, fully vaccinated and non-fully vaccinated individuals (1) are as likely to be exposed to the disease; (2) are as likely to be reported to surveillance authorities if they are hospitalized or deceased; and (3) are as likely to disclose their vaccination status if they are reported. We do not account for other potential confounding factors associated to both vaccination and the risk of hospitalization or death besides age, time and location. Next to comorbid conditions and occupation, potential confounding factors include socio-economic status [23] and differential behavior between vaccinated and non-vaccinated people. We also do not account for increasing levels of natural immunity among non-vaccinated people as time passes, which would lead to an underestimation of effectiveness of vaccines. Investigating this last point would require precise knowledge of the history of infection in hospitalized patients and in the general population. 


\section{Conclusions}

We assess real-world vaccine effectiveness against severe forms of SARS-CoV-2 infection from routine surveillance data in Switzerland, confirming the high effectiveness of mRNA vaccines from Moderna and Pfizer-BioNtech against hospitalization and death in all age groups. Effectiveness appears comparatively lower in age groups over 70, suggesting the importance of booster vaccinations. We find some evidence that the effectiveness is moderately waning over time. However, confounding by comorbid conditions and the increasing levels of natural immunity among non-vaccinated in time was not accounted for. Repeated analyses will be able to better assess waning and the effect of boosters. This approach could be implemented in most routine surveillance settings to monitor vaccine effectiveness in real time.

\section{References}

[1] F. P. Polack et al., "Safety and efficacy of the bnt162b2 mRNA covid-19 vaccine," New England Journal of Medicine, 2020.

[2] L. R. Baden et al., "Efficacy and safety of the mRNA-1273 sars-cov-2 vaccine," New England Journal of Medicine, vol. 384, no. 5, pp. 403-416, 2021.

[3] S. J. W. Evans and N. P. Jewell, "Vaccine effectiveness studies in the field," New England Journal of Medicine, vol. 385, no. 7, pp. 650-651, 2021, doi: 10.1056/NEJMe2110605.

[4] M. K. Patel et al., "Evaluation of post-introduction covid-19 vaccine effectiveness: Summary of interim guidance of the world health organization," Vaccine, 2021.

[5] N. Dagan et al., "BNT162b2 mRNA covid-19 vaccine in a nationwide mass vaccination setting," New England Journal of Medicine, vol. 384, no. 15, pp. 1412-1423, 2021, doi: 10.1056/NEJMoa2101765.

[6] B. Nunes et al., "MRNA vaccine effectiveness against covid-19-related hospitalisations and deaths in older adults: A cohort study based on data linkage of national health registries in portugal, february to august 2021," Eurosurveillance, vol. 26, no. 38, p. 2100833, 2021.

[7] J. L. Bernal et al., "Effectiveness of the pfizer-biontech and oxford-astrazeneca vaccines on covid-19 related symptoms, hospital admissions, and mortality in older adults in england: Test negative case-control study," bmj, vol. 373, 2021.

[8] E. Kissling et al., "Vaccine effectiveness against symptomatic sars-cov-2 infection in adults aged 65 years and older in primary care: I-move-covid-19 project, europe, december 2020 to may 2021," Eurosurveillance, vol. 26, no. 29, p. 2100670, 2021.

[9] W. H. Organization, "Evaluation of covid-19 vaccine effectiveness," INTERIM GUIDANCE, 2021.

[10] C. Farrington, "Estimation of vaccine effectiveness using the screening method," International journal of epidemiology, vol. 22, no. 4, pp. 742-746, 1993. 
[11] C. Mazagatos et al., "Effectiveness of mRNA covid-19 vaccines in preventing sars-cov-2 infections and covid-19 hospitalisations and deaths in elderly long-term care facility residents, spain, weeks 532020 to 13 2021," Eurosurveillance, vol. 26, no. 24, p. 2100452, 2021.

[12] S. van Buuren and K. Groothuis-Oudshoorn, "mice: Multivariate imputation by chained equations in r," Journal of Statistical Software, vol. 45, no. 3, pp. 1-67, 2011,Available: https://www.jstatsoft.org/v45/i03/

[13] S. Y. Tartof et al., "Effectiveness of mRNA bnt162b2 covid-19 vaccine up to 6 months in a large integrated health system in the usa: A retrospective cohort study," The Lancet, vol. 398, no. 10309, pp. 1407-1416, 2021.

[14] W. H. Self et al., "Comparative effectiveness of moderna, pfizer-biontech, and janssen (johnson \& johnson) vaccines in preventing covid-19 hospitalizations among adults without immunocompromising conditions-united states, march-august 2021," Morbidity and Mortality Weekly Report, vol. 70, no. 38, p. 1337, 2021.

[15] J. Lopez Bernal et al., "Effectiveness of covid-19 vaccines against the b. 1.617. 2 (delta) variant." N Engl J Med, pp. 585-594, 2021.

[16] K. Bruxvoort et al., "Effectiveness of mRNA-1273 against delta, mu, and other emerging variants," medRxiv, 2021.

[17] A. J. Pollard and E. M. Bijker, "A guide to vaccinology: From basic principles to new developments," Nature Reviews Immunology, vol. 21, no. 2, pp. 83-100, 2021.

[18] S. N. Crooke, I. G. Ovsyannikova, G. A. Poland, and R. B. Kennedy, "Immunosenescence and human vaccine immune responses," Immunity \& ageing, vol. 16, no. 1, pp. 1-16, 2019.

[19] Y. Goldberg et al., "Waning immunity after the bnt162b2 vaccine in israel," New England Journal of Medicine, 2021.

[20] H. Chemaitelly et al., "Waning of bnt162b2 vaccine protection against sars-cov-2 infection in qatar," New England Journal of Medicine, 2021.

[21] E. S. Rosenberg et al., "Covid-19 vaccine effectiveness in new york state," New England Journal of Medicine, vol. 0, no. 0, p. null, doi: 10.1056/NEJMoa2116063.

[22] P. Nordström, M. Ballin, and A. Nordström, "Effectiveness of covid-19 vaccination against risk of symptomatic infection, hospitalization, and death up to 9 months: A swedish total-population cohort study," Hospitalization, and Death Up to, vol. 9, 2021.

[23] J. Riou et al., "Socioeconomic position and the covid-19 care cascade from testing to mortality in switzerland: A population-based analysis," The Lancet Public Health, vol. 6, no. 9, pp. e683-e691, 2021. 